\title{
A Study on Career Indecision and College Students' Mobile Phone Addiction Tendency
}

\author{
Shu Cai, Guifang Fu * \\ Department of Applied Psychology, Guangdong University of Foreign Studies, Canton, China
}

*Corresponding Author: Guifang Fu, Department of Applied Psychology, Guangdong University of Foreign Studies, Canton, China

\begin{abstract}
This paper aims to explore the situation and relationship of College Students' career indecision and mobile phone addiction. The results showed that: (1) the detection rate of College Students' mobile phone addiction was $37.7 \%$. There were significant differences in gender variables, and the degree of mobile phone addiction of girls was significantly higher than that of boys ; (2) The career indecision level of college students was medium. The level of career indecision of non-only children was significantly higher than that of only children; (3) Career indecision was positively relationship with college students' mobile phone addiction. The results of this study provide the basic situation and differences of College Students' career indecision and mobile phone addiction. It has important theoretical and practical significance to provide several supports for vocational education and mental health education in Colleges and universities.
\end{abstract}

Keywords: career indecision; Mobile phone addiction; college student; obtain employment

\section{INTRODUCTION}

People's production and life are increasingly dependent on mobile phones. As a group of college students who use mobile phones heavily, the impact of mobile phone dependence (MPD) is more prominent. Mobile phone addiction, also known as mobile phone syndrome, is similar to addictive behavior. It refers to a pathological behavior in which individuals cannot control their impulse to use mobile phones, have obvious withdrawal symptoms, and damage their physiological, psychological, and social functions. Mobile phone addiction has obvious external manifestations. We can judge whether an individual has mobile phone addiction from the frequency, duration, intensity of using mobile phone, and whether it has a negative impact on his body, mind, and life. In daily life, the most obvious symptom of mobile phone addiction is "the machine does not leave the hand". When the individual is cut off from the mobile phone, the individual will become restless.

Fu Guifang et al. (2015) found that the detection rate of College Students' mobile phone addiction was $33.06 \%$, and college students with mobile phone addiction had varying degrees of mental health problems. College Students' mobile phone addiction will hinder the formation of their correct thinking concept, lead to the decline of physical function, the increase of psychological problems and the weakening of cognitive ability. Mobile phone addiction is a high-risk factor for poor sleep quality and psychological problems of college students (Zhang Ling et al., 2017). Relevant studies have pointed out that mobile phone addiction has a negative predictive effect on subjective well-being (Wang Yueqin and Zhang Yu, 2015). College students' mobile phone addiction seriously affects classroom learning efficiency (XuGuocheng et al., 2014). Mobile phone addiction can distract students' attention, increase students' depression and further affect their academic performance (SEO, D.G., et al., 2016). Mobile phone addiction will also affect college students' future college life. Some studies have shown that freshmen with high degree of mobile phone addiction will have poor adaptation to college life after becoming sophomores. There is a significant negative correlation between mobile phone addiction and college adaptation. However, sophomores with low college adaptation will have poor mental health status (i.e. subjective well-being, anxiety and depression) after being promoted to sophomores. Mobile phone addiction plays an intermediary role between college adaptation and mental health status (Guohua Zhang, Xue Yang, et al, 2020). Mobile phone addiction should be paid attention to by schools, parents and college students themselves, Exploring the mechanism of mobile phone addiction is helpful to provide enlightenment for the intervention of mobile phone addiction. 
Career consolidation is the primary development task that individuals need to complete in their early adulthood. If individuals cannot make a smooth transition at this stage, they are prone to problems such as lack of goals and anxiety. Eriksson pointed out in his psychosocial development stage theory that identity and identity confusion are the crisis that teenagers must experience in the transition from adults. The resolution of the crisis is regarded as the symbol of teenagers' completion of identity transformation, that is, the completion of self-identity. Previous studies have proved that there is a significant negative correlation between self-identity and college students' mobile phone addiction, and individuals with high self-identity have a lower degree of mobile phone addiction (Wu Zhihui, Chen Junhan, 2017; Li Zijun, 2016; Chai Xiaoyun, KeYuye, 2015). Career choice is a task that individuals must complete in the crisis of self-identity. At this stage, individuals are in the state of career indecision. They have not formed a stable and lasting commitment to what they want to do in the future, and the individuals with career indecision have not completed the integration of selfidentity. Previous studies have shown that the higher the identity status, the higher the score of career exploration and the lower the score of career decision-making difficulty (Li Wendao et al., 2007). Based on the above, the author can't help but wonder about the relationship between career indecision and mobile phone addiction.

Career indecision, also known as career indecision, refers to an individual's failure to choose the profession or career he wants to engage in. This concept was first proposed by Campbell (1981) and then widely used in career counseling, career decision-making, counseling and other related fields. Career indecision is one of the common problems for college students to go to psychological counseling centers for help. The research of career indecision has experienced a development from shallow to deep. At first, researchers only focused on the differences between career resolution and career resolution and the development of measurement tools. Later, researchers slowly turned to the discussion of possible causes and influencing factors of career resolution. At this stage, scholars realized that career resolution and career resolution were not binary opposites, but developed a multidimensional typology. For example, Larsen (1988) divided career indecision into four types: (1) unplanned escape ( 2) Knowledgeable but hesitant ( 3) Confident but lack of information (4) Lack of information. At the same time, researchers also realize that career indecision is not completely a disease, but a necessary stage for individuals to explore their careers. Some researchers divide career indecision into two kinds: one is developmental career indecision, that is, the normal of development; The other is indecisive career indecision, which is persistent and may be related to personality traits.

Scholars have less research on the relationship between career indecision and mobile phone addiction, because career indecision belongs to a type of career decision-making difficulties, and career indecision and self-identity have some commonalities. Moreover, mobile phone addiction is related to Internet addiction, problematic Internet use and excessive mobile phone use. Therefore, this study theoretically combs and reviews the correlation between career indecision and mobile phone addiction from the above aspects, and carries out empirical research.

Individuals with high level of career indecision are generally in the stage of self-identity diffusion or delay. Domestic studies have found that there is a significant negative correlation between College Students' self-identity and mobile phone addiction (Li Zijun, 2016). There is a significant difference in self-identity between high mobile phone addiction group and low mobile phone addiction group (Wu Zhihui, Chen Junhan, 2017). Diffusion style can positively predict mobile phone addiction through self-control (Chai Xiaoyun, KeYuye, 2015). Liu Ding and Lu Ning (2017) found that career decision-making difficulties can positively predict Internet addiction. College students' career decision-making difficulties and Internet addiction may be the common result of habitually using immature defense methods to avoid failure. Li Xiao, Yu Juan et al. (2019) found that college students with serious network dependence have weak professional ability, weak interpersonal relationship, and low sense of self-efficacy, which leads to college students' career decision-making difficulties. TianXiaoyan (2021) et al. Found that whether the parental rearing style is positive or not will positively affect college students' career decision-making. At the same time, some domestic scholars have studied the relationship between career planning and mobile phone addiction. The research found that the incidence of mobile phone addiction behavior of students who have carried out career planning is significantly lower than that of students who have not carried out career planning (Chen Peibo et al., 2016). Foreign studies have found that career indecision can significantly positively predict problem network use, and individuals with high level of career indecision are more likely to 
have problem network use (Lehmann \&Konstam, 2011). Stelianarizeanu (2013) found that individuals in the state of identity delay and diffusion are more prone to pathological gambling addiction. At the same time, low self-directed ability is a good predictor of problem network use. The worse the selfdirected ability is, the more likely it is to have problem network use behavior (Montag, C., et al, 2010). The above studies show that career indecision may be related to mobile phone addiction.

In the current clinical work, mobile phone addicted college students often complain about career problems, such as lack of career goals, confusion about the future and lack of interest in their major. Mobile phone addicted college students are often used as the reasons for their mobile phone addiction. Lehmann and Konstam found that there is a significant positive correlation between career unresolved problems and Internet use. Liu Ding and Lu Ning (2017) found that, there is a significant positive correlation between career decision-making difficulties and college students' Internet addiction. Career decision-making difficulties can significantly predict Internet addiction, and career indecision is a manifestation of career decision-making difficulties. Combined with contemporary college students' use of mobile phones, they mainly use their network functions, and existing studies have proved that mobile phone addiction is positively correlated with internet addiction ( $\mathrm{Li}$ Yuanwen, 2013), Therefore, we can speculate that career indecision may be the cause of College Students' mobile phone addiction.

Previous studies often focused on the adverse side of mobile phone addiction, and most of them focused on more stable or external factors such as personality traits and peer relationship, but less on the positive side of mobile phone use for individuals and the impact of individual events in the current state on College Students' mobile phone addiction. For college students in their early adulthood, mobile phones are an important tool for individuals to obtain information, make friends and entertainment. Understanding the role of individual life events on College Students' mobile phone addiction is helpful to provide substantive enlightenment for solving related problems. On the basis of reviewing a large number of literature and relevant theories, this study attempts to explore the relationship between career indecision and mobile phone addiction from the perspective of career, and assumes that career indecision and mobile phone addiction have a positive predictive effect.

Theoretically, there is no scholar at home and abroad to explore the causes of mobile phone addiction from the perspective of career. Based on a large number of literature review, this study explores the mechanism of mobile phone addiction from a new perspective through empirical analysis. In a practical sense, this study is conducive to provide enlightenment for the intervention of mobile phone addiction, and provide new ideas for school workers, psychological workers, and college students to prevent and treat mobile phone addiction.

\section{METHOD}

\subsection{Subjects}

The questionnaire was distributed in the form of wechat forwarding to collect data. A total of 605 questionnaires were sent and 584 valid questionnaires were recovered, with an effective recovery rate of $96.5 \%$. A total of 220 male subjects were selected, 364 were female, among which only child accounted for $44.3 \%$ of the total number, and registered residence accounted for $62.8 \%$ of the total number. The questionnaires were distributed to different majors from freshmen to seniors. The freshmen recovered 220 copies, accounting for $37.7 \%$, and the sophomores recovered 134 copies, accounting for $22.9 \%$. 113 copies were recovered in junior college, accounting for 19.3\%, and 117 copies were recovered in Senior College, accounting for 20\%. 121 subjects (20.7\%) majored in literature and history, 241 subjects $(41.3 \%)$ in science and engineering, 176 subjects $(30.1 \%)$ in economics and management, and 46 subjects $(7.9 \%)$ in others.

\subsection{Measures}

\subsubsection{The college students' mobile phone addiction tendency scale}

The measurement adopts the college students' mobile phone addiction tendency scale (mpats) compiled by XiongJie et al. (2012). The questionnaire contains 16 items, for example, "I often fear that my mobile phone will turn off automatically". All items are scored by 5 points, from 1 (completely inconsistent) to 5 (fully consistent). The scores of each item can be summed up, with a total score of 80 points. The higher the score, the more serious the degree of mobile phone addiction. 
The scale is widely used in domestic research. The research shows that the questionnaire has good reliability and validity. In this study, the Cronbach $\alpha$ of the scale is 0.871 .

\subsubsection{Career decision scale}

The career indecision subscale in the career decision scale (CDS) was used to evaluate the individual's career indecision level. The scale contains 16 items. For example, "I can't make a career decision right now because I don't know what ability I have". All items are scored by 7 points, of which 1 means completely inconsistent, 4 means uncertain and 7 means fully consistent. The higher the score, the higher the individual's career indecision level. The scale is widely used by researchers at home and abroad, and the scale is explained by the total score. In this study, the Cronbach $\alpha$ of the scale is 0.881 .

\section{RESUlT}

\subsection{Common Method Bias Tests}

Common method Bias Tests refers to the artificial covariance between prediction variables and standard variables caused by the same data source or rater, the same measurement environment, project context and the characteristics of the project itself (Zhou Hao, long Lirong, 2004). Since this study uses self-report method to collect data, it may lead to common method deviation effect. This study used procedural methods to control, including: (1) all questionnaires were anonymous (2) The scale or questionnaire used has high reliability and validity, so as to reduce or avoid the systematic error of measurement as much as possible(3) Some items in the questionnaire use reverse scoring (4) The data were collected in the form of wechat forwarding. The subjects came from different schools and regions to increase the difference of source space. In addition, after data collection, Harman single factor test was further used to diagnose the common method deviation. The results showed that the eigenvalues of 12 factors without rotation were greater than 1 , and the variation explained by the first factor was $19.90 \%$, less than the critical standard of $40 \%$. This shows that the deviation of the common method is not obvious.

\subsection{Situation of College Students' mobile phone addiction}

As shown in Table 1, the detection rate of College Students' mobile phone addiction is $37.7 \%$. Table 2 shows that the average total score of College Students' mobile phone addiction is 44.47 , which is close to the addiction level. The total score of the questionnaire is greater than or equal to 48, which can be regarded as mobile phone addiction.

Table1. Detection rate of College Students' mobile phone addiction

\begin{tabular}{|c|c|c|c|c|}
\hline & Total & critical value & frequency & Detection rate (\%) \\
\hline phone addiction & 584 & $\geq 48$ & 220 & 37.7 \\
\hline
\end{tabular}

Table 2 shows that college students' mobile phone addiction is generally at a high level, and their withdrawal behavior is the highest, indicating that when mobile phone addicted college students do not use mobile phones, they will have bad emotions, unable to study normally and other bad behaviors to a certain extent.

Table2. Descriptive statistics of College Students' mobile phone addiction

\begin{tabular}{|c|c|c|c|c|}
\hline & Minimum & Maximum & Mean & SD \\
\hline withdrawal symptoms & 6 & 30 & 18.66 & 4.24 \\
\hline Salience & 4 & 20 & 9.80 & 3.09 \\
\hline social comfort & 3 & 15 & 8.24 & 2.56 \\
\hline Mood change & 3 & 15 & 7.77 & 2.46 \\
\hline Total score & 16.00 & 80.00 & 44.47 & 9.85 \\
\hline
\end{tabular}

\subsection{Difference test of College Students' mobile phone addiction in demographic variables}

T-test registered a significant difference in the variables of gender, whether it is only child or family registered residence type, as shown in Table 3. T test showed that there were significant differences in the gender of mobile phone addiction. The results showed that there were significant differences in gender variables of College Students' mobile phone addiction $(t=-2.57, \mathrm{P}<0.01)$. Among them, the score of women's mobile phone addiction was significantly higher than that of men. Whether mobile phone addiction is not affected by the single child or household registered residence type is 
significant. F test showed that there were no significant differences in mobile phone addiction among grades and disciplines.

Table3. $t$-test of mobile phone addiction on demographic variables

\begin{tabular}{|c|c|c|c|c|c|c|}
\hline & & Number & mean & SD & $F$ & $t$ \\
\hline Gender & Male & 220 & 43.13 & 9.49 & \multirow[t]{2}{*}{1.84} & \multirow[t]{2}{*}{$-2.57 * *$} \\
\hline & Femal & 364 & 45.28 & 9.99 & & \\
\hline whether only one-child & Yes & 259 & 43.66 & 9.39 & \multirow[t]{2}{*}{2.49} & \multirow[t]{2}{*}{1.79} \\
\hline & No & 325 & 45.12 & 10.18 & & \\
\hline census register type & Rural & 217 & 44.23 & 9.46 & \multirow[t]{2}{*}{0.89} & \multirow[t]{2}{*}{0.45} \\
\hline & Urban & 367 & 44.61 & 10.09 & & \\
\hline
\end{tabular}

Note: ** indicates significant at the level of 0.01

\subsection{Current situation of College Students' career}

Table 4 shows that the average total score of College Students' career indecision is 66.69, with a medium level.

Table4. Current situation of College Students' career

\begin{tabular}{|l|l|l|l|l|l|}
\hline & Number & Minimum & maximum & mean & SD \\
\hline Career Indecison score & 584 & 16 & 112 & 66.69 & 14.25 \\
\hline
\end{tabular}

\subsection{Relationship between career indecision and mobile phone addiction}

The results showed that there was a significant positive correlation between career indecision and mobile phone addiction and its dimensions $(\mathrm{P}<0.01)$, as shown in Table 5. It shows that the higher the degree of career indecision of college students, the greater the possibility of their mobile phone addiction.

Table5. Correlation analysis between College Students' career indecision and mobile phone addiction

\begin{tabular}{|l|l|l|l|l|l|l|}
\hline & $\begin{array}{l}\text { Career } \\
\text { Indecison }\end{array}$ & $\begin{array}{l}\text { withdrawal } \\
\text { symptoms }\end{array}$ & Salience & social comfort & $\begin{array}{l}\text { Mood } \\
\text { change }\end{array}$ & $\begin{array}{l}\text { Mobile phone } \\
\text { addiction }\end{array}$ \\
\hline $\begin{array}{l}\text { Career } \\
\text { Indecision }\end{array}$ & 1 & & & & & \\
\hline $\begin{array}{l}\text { Social } \\
\text { Anxiety }\end{array}$ & $0.36^{* *}$ & & & & & \\
\hline $\begin{array}{l}\text { withdrawal } \\
\text { symptoms }\end{array}$ & $0.35^{* *}$ & 1 & & & & \\
\hline Salience & $0.33^{* *}$ & $0.65^{* *}$ & 1 & & & \\
\hline $\begin{array}{l}\text { social } \\
\text { comfort }\end{array}$ & $0.32^{* *}$ & $0.43^{* *}$ & $0.40^{* *}$ & 1 & & \\
\hline $\begin{array}{l}\text { Mood } \\
\text { change }\end{array}$ & $0.34^{* *}$ & $0.57^{* *}$ & $0.53^{* *}$ & $0.34^{* *}$ & 1 & \\
\hline $\begin{array}{l}\text { Mobile } \\
\text { phone } \\
\text { addiction }\end{array}$ & $0.42^{* *}$ & $0.89^{* *}$ & $0.83^{* *}$ & $0.66^{* *}$ & $0.75^{* *}$ & 1 \\
\hline
\end{tabular}

Note: $* *$ indicates significant at the level of 0.01

\section{DSISSCUSSION}

\subsection{Current Situation and Demographic Differences of College Students' Mobile Phone Addiction}

The study found that the detection rate of College Students' mobile phone addiction was $37.7 \%$. Compared with previous studies, college students' mobile phone addiction shows a serious trend. With the rapid development of science and technology, the production cost of mobile phones has decreased, and mobile phones have entered the homes of ordinary people. In addition, with the increasing enrichment of mobile phone functions and the portability of mobile phones, mobile phones have increasingly replaced computers, televisions and tablets and become the basis for the interconnection of all things. College students are more likely to accept new things, and mobile phones can meet the social, information search, entertainment and other needs of college students. It is normal for college 
students to "stay on the machine and stay on the machine". College Students' rational use of mobile phones is conducive to their study and life, but excessive use of mobile phones will affect their learning efficiency, lead to a decline in academic performance, and seriously affect their physical and mental health. The problem of College Students' mobile phone addiction deserves the attention of schools and relevant parties.

T-test mobile phone addiction registered a significant difference in gender variables. The degree of mobile phone addiction was significantly higher than that of boys, but the influence of grade, specialty, subject only, child only and family registered residence on $t$ addiction was not significant. In the review part, it is mentioned that the previous research conclusions are inconsistent in whether there are differences in gender on mobile phone addiction. In this study, gender variables have a significant impact on mobile phone addiction. The inconsistency of research conclusions reminds us whether there are other factors between gender differences affecting the degree of mobile phone addiction, such as whether the different ways of pressure elimination and entertainment between men and women (such as boys prefer to play online games) will affect the degree of mobile phone addiction, or whether there is a substitution relationship between mobile phone addiction and other addiction ways (such as game addiction). Huang Hai et al. (2014) found that boys scored higher on Internet dependence than girls, while girls scored higher on mobile phone addiction than boys. At the same time, in this study, it was found that girls' social anxiety level was significantly higher than boys, and men and women had different coping strategies in the face of anxiety, These may be the reason why the degree of mobile phone addiction of girls is higher than that of boys. Mobile phone addiction is mobile phone addiction, but the variables such as grade, household registered residence, place of origin, and subject of professional subjects are not significant.

\subsection{The Current Situation of College Students' Indecision Career and its Demographic Differences}

The study found that the career level of college students is medium. Generally, career indecision is a state that an individual must experience in the transitional stage of obtaining adult identity. The length of this stage and the level of career indecision vary from person to person and are affected by many factors, such as family factors (family economic level, parents' educational level, etc.), personal personality characteristics (such as perfectionism), etc. The career indecision subscale used in this study is a one-dimensional scale, which can generally be used to understand the individual's career indecision level in clinical practice. However, there is no clear standard on how much higher the score of career indecision can be defined as career indecision.

The results of t-test show that there is a significant difference in the variable of "whether the only child or not", and the level of career indecision of non only children is significantly higher than that of only children. Parents, as important others of their children, are important resources for individuals to seek support and suggestions in the process of career choice. They have an important impact on their children's career choice. Sometimes parents can even directly determine their children's career choice. Parents' participation in their children's career planning will reduce the degree of individual career indecision (QiuLilin, 2014). Whether the only child will affect the amount of resources individuals get from their parents, and the only child can often get more attention, support and suggestions from their parents, which may be the reason why the level of career indecision of non-only children is significantly higher than that of only children. However, the grades, occupation, gender, and household registered residence have no significant impact on College Students' career indecision. Generally, we believe that the career indecision level of majors with good employment prospects should be relatively low, but no difference in the career indecision level between the disciplines of the majors is found in this study. The reasons may be as follows: (1) the disciplines of the majors in this study have a large scope, the same discipline contains more majors, and the disciplines of each major include both popular majors, It also includes popular majors ( 2) Under the severe employment situation, there is no significant difference in the level of career indecision among the disciplines to which the major belongs. Similarly, intuitively, we would think that there should be significant differences in the level of career indecision between grades. In fact, career suspense is a developmental norm that runs through the University and even the early stage of employment. Many foreign researchers study the career suspense of individuals in their infancy (18-29 years old). Everyone must experience a state of career suspense before determining a stable career. In previous 
studies, the impact of grade on career suspense is not deterministic. For example, Li Leiqiong (2007) found that there are differences in developmental suspense among college students of all grades, but there is no significant difference in emotional disorder career suspense, and Scheffe's post comparison difference in developmental career suspense is not significant, Yu Shujun (2008) found that there was no significant difference in college students' career indecision level among grades. Since this study uses a single-dimensional career indecision, whether there are differences in the specific types of career indecision in grades needs further research.

\subsection{The Relationship between College Students' Career Indecision and Mobile Phone Addiction}

he results of this study show that college students' career indecision has a direct positive effect on mobile phone addiction, and indirectly affects mobile phone addiction through social anxiety. The results meet the research expectations. Career decision-making is a complex and challenging task. For college students in their infancy, it is an inevitable development task. When faced with career decision-making difficulties, individuals may be more inclined to pay attention to themselves, and may project the emotions generated by such decision-making difficulties into interpersonal communication, which will increase their social anxiety. Individuals in their early adulthood have a sense of independence. They will try to make decisions independently, but they do not fully have this ability. Mobile phones can just meet the needs of college students to seek private space, collect information and obtain virtual social support. To some extent, this is conducive to improving college students' career decision-making and facilitating them to make career decisions. This suggests that mobile phone addiction may be a form of seeking external information in the indecision state of occupation. However, due to the lack of richness and effectiveness of information resources on the network, it may cause individual cognitive overload, which is not conducive to individual career decision-making. In this sense, mobile phones are more likely to be a "harbor" for individuals with high professional level to seek support and protection. In addition, career decision-making itself is not an overnight process, and the persistence of this process is more likely to make college students form mobile phone addiction. Mobile phone addiction may also affect career decision-making and delay their career positioning, which needs further research. It should be pointed out that career indecision is not completely a disease. Among college students, it is a developmental norm and the only way for individuals to change their roles to adults. On this road, we can appropriately actively guide college students' career decision-making, so as to make college students become individuals with goals and self-control, so as to reduce the degree of mobile phone addiction.

\section{CONCLUSION}

The main conclusions are as follows: (1) the detection rate of College Students' mobile phone addiction is $37.7 \%$, and there is a significant difference in gender variables $(\mathrm{t}=-2.57, \mathrm{P}<0.01)$. The degree of mobile phone addiction of girls is significantly higher than that of boys (2) The level of career indecision of college students is medium $(\mathrm{M}=66.69, \mathrm{SD}=14.25)$. There is a significant difference in whether they are the only child or not $(\mathrm{t}=-3.30, \mathrm{P}<0.01)$. The level of career indecision of non only children is significantly higher than that of the only child (3) Career indecision has a significant direct and positive predictive effect on College Students' mobile phone addiction.

This study proves that career problems represented by career unresolved do have an impact on mobile phone addiction. Mobile phone addiction may be an immature defense mechanism for individuals to avoid career decision-making. This suggests that career counseling may improve college students' mobile phone addiction, but in clinical work, psychologists should pay attention to the psychological motivation of mobile phone addicts' excessive use of mobile phones, understand that mobile phone use has a special defense function for this group, and formulate more effective intervention strategies. In view of the serious situation of College Students' mobile phone addiction, the school can also strengthen the guidance of college students, set up effective career counseling courses, help college students set correct and feasible goals and establish correct time management concepts, and reduce the degree of mobile phone addiction.

With the rapid development of mobile phone intelligence and Internet, whether mobile phone addiction will further spread in the population deserves follow-up attention. Most of the existing research on mobile phone addiction is based on the sample of college students. Whether different ages, occupations and social strata will affect mobile phone addiction needs to be further discussed. This study proves that there is a certain relationship between career indecision and mobile phone 
addiction, but this study does not distinguish between developmental indecision and indecisive career indecision. The former is the normal development, and the latter is related to personality traits. Distinguishing the different effects of the two on mobile phone addiction is of great significance to the intervention of clinical work on mobile phone addiction. In addition, the longitudinal research on career indecision and mobile phone addiction needs to be in-depth. If the level of career indecision decreases with the development of time, excluding the influence of other factors, it will reduce the degree of mobile phone addiction, which will more strongly prove the relationship between career indecision and mobile phone addiction.

\section{ACKNOWLEDGEMENT}

This work was supported by The National Social Science Fund of China [grant number 16BSH094]

\section{REFERENCES}

[1] FU Guifang, LI Xinyao. Relationship between College Students' Mobile Phone Addiction and Mental Health [J]. Journal of Changchun University, 2015, 25 (12): 52-55.DOI: 10.3969/j.issn.10093907.2015.06.013

[2] FU Guifang, LI Xinyao, XU Zhukun, WEI Xianjun. Investigation on College Students' Mobile Dependence [J]. Journal of Higher Education, 2016 (01): 65-66+68. DOI: CNKI:SUN:GJXK.0.2016-01030

[3] ZHANG Ling, ZHU Jie, WANG Jianming. Effects of Mobile Dependence Phenomenon on Contemporary College Students' Sleep and Mental Health [J]. Journal of Public Health and Preventive Medicine, 2017, 28 (02): 108-111. DOI: CNKI:SUN:FBYF.0.2017-02-030

[4] WANG Yueqin, ZHANG Yu. Relationship between College Students' Mobile Dependence and Comprehending Social Support and Their Subjective Well-being [J]. Chinese Mental Health Journal, 2015, 29 (11): 868-873. DOI: 10.3969/j.issn.1000-6729.2015.11.013

[5] XuGuocheng, Huang Li, Wei Lili, Zhu Dandan. Study on the relationship between College Students' mobile phone addiction and classroom learning efficiency [J]. Journal of Zhejiang University of technology, 2014,32 (12): 535-538 + 543

[6] Zhang Yan. Investigation and Research on College Students' mobile phone addiction [J]. Modern women (late), 2013 (11): 173

[7] LI Yuanwen. Research on the Characteristics of and Relationship between Peer Attachment and Loneliness in Teenagers Addicted to Internet and Mobile Phone [D]. Central China Normal University, 2013. DOI: 10.7666/d.Y2504043

[8] XIONG Jie, ZHOU Zongkui, CHEN Wu, YOU Zhilin, ZHAI Ziyan. Compilation of Mobile Phone Addiction Tendency Scale for College Students [J].Chinese Mental Health Journal, 2012, 26 (03): 222 $\neg 225$. DOI: $10.3969 /$ j.issn.1000-6729.2012.03.013

[9] LIU Xin, CHEN Jing, LEI Wei, LIANG Xuemei, LIU Kezhi. Relationship between College Students' Mobile Phone Addiction and Social Anxiety: Mediating Roles of Interpersonal Relationship [J]. Journal of Southwest Medical University, 2017, 40 (04): 392-396. DOI: 10.3969/j.issn.2096-3351.2017.04.017

[10] HU Shanshan. Research on the Relationship among College Students' Control Sources, Social Anxiety and Mobile Phone Addiction Tendency [D]. Beijing Institute of Technology, 2015. URL: https://kns.cnki. net $/ \mathrm{kcms} / \mathrm{detail} / \mathrm{detail}$. aspx ?dbcode=CMFD\&dbname $=$ CMFD201502\&filename $=1015029672 . \mathrm{nh} \& \mathrm{v}=\mathrm{Ia} \% 2$ 5mmd2BaZDsJwB2IQdFUHN06uY0SDZ58JxPkYjXIZNgDhT9pzzcaJnzihDG09PUDhF0W

[11] WANG Huan, HUANG Hai, WU Heming. Relationship between College Students' Personality Characteristics and Mobile Dependence: Mediating Roles of Social Anxiety [J]. Chinese Journal of Clinical Psychology, , 22 (03): 447-450. DOI:CNKI:SUN:ZLCY.0.2014-03-015

[12] Chai Xiaoyun, KeYuye. The relationship between identity style, self-control, and mobile phone addiction among college freshmen [J]. Chinese Journal of health psychology, 2015,23 (06): 881-885

[13] Li Wendao, Zou Hong, Zhao Xia. The relationship between College Students' identity and career exploration and career decision-making difficulties [J]. Psychological development and education, 2007 (02): 63-67

[14] LIU Ding, LU Ning. Relationship between Achievement Motivation and College Students' Internet Addiction: the Mediating Roles of Immature Defense Methods and Difficulties in Career Decision-making [J]. Chinese Journal of Clinical Psychology, 2017, 25 (06): 1093-1097. DOI: 10.16128/j.cnki.10053611.2017.06.021

[15] LI Xiao, YU Juan, WANG Haihong, \& WANG Yajuan. The Influence of Network Dependence on College Students' Career Decision-Making Difficulty -- Mediated by the Trust of Network Information [J]. Journal of Yangzhou University (Higher Education Study Edition), 2019, 23(05), 96-99. DOI: 
10.19411/j.cnki.1007-8606.2019.05.018

[16] LIU Qingxue, YANG Yan, et al., (2017). Smartphone Addiction: Concept, Measurement, and Influencing Factors [J]. Chinese Journal of Clinical Psychology, 2017, 25(1), 82-87.DOI: 10.16128/j.cnki.10053611.2017.01.019

[17] ZOU Yan, CHEN Shanjiao, DONG Qian, WANG Hong, XIE Xin, JI Shanshan, YU De'e. Status Quo of Mobile Addiction and Its Relationship with Personality in College Students in Hainan [J]. China Journal of Health Psychology, 2017, 25 (09): 1372-1375. DOI: 10.13342/j.cnki.cjhp.2017.09.025

[18] Zhang Xi, Zhang Yan, Wu min. study on the relationship between College Students' self-control and mobile phone addiction [J]. Journal of Lanzhou Institute of education, 2017,33 (09): 158-160

[19] Wang Fuzhong. Study on the relationship between College Students' psychological capital, overall wellbeing and mobile phone addiction $[\mathrm{J}]$. Journal of Taiyuan Normal University (SOCIAL SCIENCE EDITION), 2017,16 (06): 106-108

[20] Wu Zhihui, Chen Junhan. Research on mobile phone addiction, self-identity and self-esteem of college students [J]. Journal of Hunan University of Humanities and technology, 2017,34 (05): 120-123

[21] Wu Huiliang. Investigation on the current situation of College Students' mobile phone addiction and its relationship with personality traits and coping styles [D]. Jiangxi Normal University, 2015.7,34 (05): 120123

[22] Hong Yanping, Xiao Xiaoqin. Mobile phone addiction and personality traits of college students [J]. Chinese Journal of health psychology, 2013,21 (04): 626-628

[23] Jiang Jingchuan. Research progress and Enlightenment of foreign college students' career indecision [J]. Journal of Jiangsu Normal University (PHILOSOPHY AND SOCIAL SCIENCES EDITION), 2013,39 (01): 139-144

[24] Wang Lixin. A review of anxiety theory in psychoanalysis [J]. Journal of Chengdu University (SOCIAL SCIENCE EDITION), 2003 (02): 15-17

[25] CHEN Peibo, ZHAO Yanli, ZHAO Mei. Analysis of the Influence of Career Planning on College Students' Soft Addiction Behaviors [J]. China Higher Medical Education, 2016 (04): 55-56. DOI: 10.3969/j. issn.1002-1701.2016.04.028

[26] WANG Lishen, ZHU Ruqian. Influence of Social Anxiety on College Students' Mobile Dependence and Loneliness [J].Journal of Shaoguan University, 2016, 37 (07): 122-125. DOI: 10.3969/j.issn.10075348.2016.07.027

[27] SHI Zifu, ZHENG Kaiwen, ZHANG Huimin, FANG Zhou, DING Qiaoshi. Relationship among College Students' Personality Traits, Social Anxiety and Mobile Dependence [J]. Psychological Research, 2017, 10 (01): 91-96. DOI: 10.3969/j.issn.2095-1159.2017.01.013

[28] HE Sha. Mediating Roles of College Students' Social Anxiety in Interpersonal Trust's Affecting Mobile Phone Addiction [D]. Shaanxi Normal University, 2016. URL: https://kns.cnki.net/kcms /detail/detail.aspx ddbcode=CMFD\&dbname $=$ CMFD 20 1701\&filename $=1017020480$. nh \&v=f4qL4\%25 mmd2BNymxaIhIU2Ydy0Zibq5Xq\%25mmd2BCshFK31eYE0k15oPBEqxMcqU9E7GGvmIwZS5

[29] DAI An. Research on the Relationship between College Students' Mobile Dependence and Personality and Social Anxiety [D]. Yangzhou University, 2017. DOI: CNKI:CDMD:2.1017.117676

[30] JIANG Yongzhi, WANG Haixia, BAI Xiaoli. Relationship between College Students' Social Anxiety and Mobile Internet Use: Intermediaries of Preferences of Mobile Social Network Use [J]. Journal of Guizhou Normal University (Natural Science Edition), 2016, 34 (01): 103-108. DOI: 10.3969/j.issn.10045570.2016 .01 .022

[31] Qiuhaozheng, Lin Bifang. Principle and application of structural equation model [M]. Beijing: China Light Industry Press, 2009:101

[32] Zhou Hao, long Lirong. Statistical test and control method of common method deviation [J]. Progress in psychological science, 2004 (06): 942-950

[33] WANG Huan, HUANG Hai, WU Heming. Relationship between College Students' Personality Characteristics and Mobile Dependence: Mediating Roles of Social Anxiety [J]. Chinese Journal of Clinical Psychology, , 22 (03): 447-450. DOI: CNKI:SUN:ZLCY.0.2014-03-015

[34] QIU Lilin. Career Indecision-Influence Mechanism of Personality and Decision Style [D]. Central China Normal University, 2014. DOI: CNKI:CDMD:2.1014.238788

[35] LI Leiqiong. Roles of Family Factors in College Students' Self-efficacy in Career Decision $\neg$ making and Career Indecision [D]. Jiangxi Normal University, 2007. DOI: 10.7666/d.y1223750

[36] YU Shujun. Research on the Relationship among Perfectionism, Self-efficacy in Career Decision $\neg$ making and Career Indecision [D]. Beijing Normal University, 2008. DOI: 10.7666/d.y1223750

[37] Seo, D.G., et al., Mobile phone dependency and its impacts on adolescents' social and academic behaviors 
[J]. Computers in Human Behavior, 2016. 63: p. 282-292.

[38] Yang, X., Tu, X., Ding, N., \& Lau, J. T. F. (2020). Prospective relationships between mobile phone dependence and mental health status among Chinese undergraduate students with college adjustment as a mediator. Journal of Affective Disorders, 260, 498-505.

[39] TianXiaoyan,HuangBijuan,LiHongxia,XieShaowen,AfzalKomal,SiJiwei\& $\quad$ Hu Dongmei.(2021).How Parenting Styles Link Career Decision-Making Difficulties in Chinese College Students? The Mediating Effects of Core Self-Evaluation and Career Calling . Frontiers in Psychology.

[40] Lehmann, I. S., \&Konstam, V. (2011). Growing Up Perfect: Perfectionism, Problematic Internet Use, and Career Indecision in Emerging Adults [J], 89(2), 155-162.

[41] Arnett, J. J., Žukauskienè, R., \& Sugimura, K. (2014). The new life stage of emerging adulthood at ages 18-29 years: implications for mental health [J]. The Lancet Psychiatry, 1(7), 569-576.

[42] Arnett, J. (2006). Emerging adulthood: Understanding the new way of coming of age. In J. Arnett \& J. Tanner (Eds.), Emerging adults in America: Coming of age in the 21st century (pp. 1-3) [M]. Washington, DC: American Psychological Association.

[43] Salicetia, F. (2015). New Addictions [J]. Procedia - Social and Behavioral Sciences, 191, 1639-1643.

[44] Osipow, S. H., \& Winer, J. L. (1996). The Use of the Career Decision Scale in Career Assessment [P], 4(2), 117-130.

[45] Nichols, A. L., \& Webster, G. D. (2015). Designing a brief measure of social anxiety: Psychometric support for a three-item version of the Interaction Anxiousness Scale (IAS-3) [J]. Personality and Individual Differences, 79, 110-115.

[46] Pourrazavi, S., Allahverdipour, H., Jafarabadi, M. A., \&Matlabi, H. (2014). A socio-cognitive inquiry of excessive mobile phone use [J]. Asian Journal of Psychiatry, 10, 84-89.

[47] Rizeanu, S., Pathological Gambling in Relation to Anxiety and Identity Status [J]. Procedia - Social and Behavioral Sciences, 2013. 78: p. 748-752.

[48] Montag, C., M. Jurkiewicz and M. Reuter, Low self-directedness is a better predictor for problematic internet use than high neuroticism [J]. Computers in Human Behavior, 2010. 26(6): p. 1531-1535.

[49] Prizant-Passal, S., T. Shechner and I.M. Aderka, Social anxiety and internet use - A meta-analysis: What do we know? What are we missing? [J] Computers in Human Behavior, 2016. 62: p. 221-229.

[50] Kim, E. and E. Koh, Avoidant attachment and smartphone addiction in college students: The mediating effects of anxiety and self-esteem [J]. Computers in Human Behavior, 2018. 84: p. 264-271.

Citation: Shu Cai, Guifang Fu. "A study on career indecision and college students' mobile phone addiction tendency" International Journal of Humanities Social Sciences and Education (IJHSSE), vol 8, no. 8, 2021, pp. 96-105. doi: https://doi.org/10.20431/2349-0381.0808008.

Copyright: (C) 2021 Authors. This is an open-access article distributed under the terms of the Creative Commons Attribution License, which permits unrestricted use, distribution, and reproduction in any medium, provided the original author and source are credited. 